\title{
Geografía y cambios en la calidad de vida de los argentinos: una perspectiva territorial a la luz del siglo XXI
}

Guillermo Angel Velázquez

Instituto de Geografía, Historia y Ciencias Sociales / Centro de Investigaciones Geográficas, Universidad Nacional del Centro de la Provincia de Buenos Aires, Argentina.

Recibido: 14 de noviembre de 2017. Aceptado: 23 de julio de 2018.

\begin{abstract}
Resumen
El análisis de la calidad de vida desde una perspectiva territorial requiere del relevamiento de variables significativas (la importancia consiste en su poder de discriminar las situaciones que son diferentes). Consideramos principalmente la dimensión socioeconómica (educación, salud y vivienda) y la ambiental (problemas ambientales y recursos recreativos). Desde esta perspectiva, los mapas y cuadros comparativos demuestran claramente que la situación de la población Argentina, que había mejorado muy poco entre 1991 y 2001, progresó considerablemente entre 2001 y 2010; principalmente en lo que respecta a educación y salud. El análisis territorial a escala departamental/ comunal (525 unidades) pone en evidencia estos elocuentes avances en el territorio; muy especialmente en las regiones con peor posición relativa. Esta situación se registra por primera vez desde la década del ochenta hasta la actualidad.
\end{abstract}

PALABRAS CLAVE: CALIDAD DE VIDA. ARGENTINA. DEPARTAMENTOS/COMUNAS. ÍNDICES.

\section{Geography and changes in quality of life of argentines: a territorial perspective in the light of the 21st century}

\begin{abstract}
Analysis of the quality of life requires the survey of significant variables, if we pursue a territorial perspective (their importance consists in its power to discriminate different situations). Mainly, we consider both the socioeconomic dimension (education, health and housing) and the environmental one (environmental problems and recreational resources). From this perspective, the comparative maps and tables clearly show that the situation of the argentine population, which had been poorly improved between 1991 and 2001, nevertheless had considerably progressed between 2001 and 2010; mainly in education and health dimensions. Territorial analysis, at departmental/communal level (525 units), reveals these eloquent advances in the territory, especially in the regions with the worst relative position. This is the first time we register this situation since the decade of the eighties until the present time.
\end{abstract}




\section{Introducción}

Mucho hemos trabajado este tema para Argentina y por eso tenemos a nuestra disposición una serie histórica que permite ver evoluciones en la calidad de vida de los argentinos basándonos en estadísticas comparables, más allá de percepciones o expresiones de deseos de los residentes y agentes hegemónicos que intentan imponer imágenes a partir de un supuesto consenso. Por otra parte, se considera a todo el territorio nacional, pero recurriendo a la mínima unidad posible en cuanto a la información pertinente (cada uno de los 510 partidos/departamentos y las 15 comunas de la Ciudad Autónoma de Buenos Aires -CABA-), para exponer aquellos factores que más discriminan y la posición relativa de cada unidad con respecto a las demás para cada período y territorio; lo cual consideramos un insumo muy valioso para el diagnóstico y la gestión.

Nos orientamos preferentemente hacia métodos cuantitativos; esto es porque el logro de mejores Índices de Calidad de Vida (ICV) debe ser una guía para la ejecución de las políticas públicas de salud, vivienda, educación, medio ambiente y cultura, independientemente de si los habitantes o los sectores hegemónicos creen que es alta o baja desde sus propios intereses o percepción.

Nuestro índice opera con límites máximos y mínimos; y si bien, los lugares que poseen peor calidad de vida coinciden mayormente con los lugares de mayor pobreza, esto último no es nuestro objeto de estudio. La calidad de vida es una medida de logro respecto de un nivel establecido como óptimo, teniendo en cuenta dimensiones socioeconómicas y ambientales que dependen de la escala de valores prevalecientes en la sociedad y que varían en función de las expectativas de progreso histórico (Velázquez, 2001).

La formulación de un índice de calidad de vida que sirva a toda realidad mundial o a todo momento histórico no es cuestión totalmente resuelta. Los trabajos de: Barclay, 1962; Bolsi, et al. 2006; Celemín, 2007; Connerly et al., 1985; Delgado et al., 1996; Estés, 1993; Fernández-López et al., 2010; Friel et al., 2011; Giusti, 2007; Halperín, 1994; Lo Vuolo et al., 1992; Longhi et al., 2013; Lucero et al., 2007; McCann, 2004; Mikkelsen et al., 2013; Olave et al., 1995; Rofman, 1999; Rogerson, 1999; Santos, 1979; Sterimberg et al., 2004; Tanguay et al., 2010; Torrado, 2007; Wilkins, 2003, entre otros, contribuyen a generar un marco que debemos adaptar a la escala de valores y a la disponibilidad de datos en Argentina; por tanto, este índice está en permanente construcción a partir de diversas validaciones in situ realizadas luego de cada medición. En el índice utilizado en 2001 se incorporaron tan sólo los problemas ambientales como indicador negativo. Posteriormente, el trabajo de campo, en el marco de nuevas discusiones teóricas y metodológicas, nos llevó a incorporar el impacto de lo que denominamos recursos recreativos de base natural y recursos recreativos socialmente construidos, que influyen significativamente en la calidad ambiental y, por ende, en la calidad de vida de la población. Esta actualización de dimensiones y variables contribuye al propósito de reflejar y discriminar mejor las diferencias presentes, aunque obliga a efectuar adaptaciones para poder realizar análisis temporales comparativos. 
La ponderación de cada variable del ICV está explicada en detalle en Velázquez (2016) y puede resumirse en el Cuadro 1.

Cuadro 1. Peso de variables del Índice de Calidad de Vida (ICV) 2010. Fuente: las fórmulas matemáticas y cálculos según puntajes omega se encuentran en Velázquez (2016) y Velázquez y Celemín (2013).

\begin{tabular}{|c|c|c|c|c|}
\hline & & $\begin{array}{c}\text { Variable(s): } \\
(6+11)\end{array}$ & $\begin{array}{c}\text { Peso } \\
\text { parcial (\%) }\end{array}$ & $\begin{array}{c}\text { Peso } \\
\text { Total (\%) }\end{array}$ \\
\hline \multirow{7}{*}{ 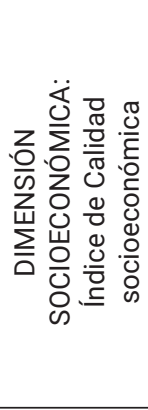 } & \multirow{2}{*}{ Vivienda } & Sin retrete (SE1) & 10 & 20 \\
\hline & & Hacinamiento (SE2) & 10 & \\
\hline & \multirow{2}{*}{ Salud } & TMI (SE3) & 10 & 20 \\
\hline & & Sin obra social (SE4) & 10 & \\
\hline & \multirow{2}{*}{ Educación } & $\begin{array}{c}\text { Educación menor a Primaria } \\
\text { (SE5) }\end{array}$ & 10 & 20 \\
\hline & & $\begin{array}{l}\text { Educación universitaria o } \\
\text { superior completa (SE6) }\end{array}$ & 10 & \\
\hline & & & Total & 60 \\
\hline \multirow{4}{*}{ 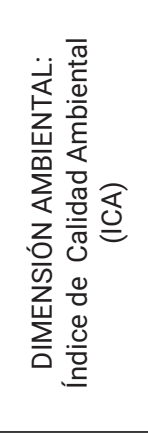 } & $\begin{array}{c}\text { Recursos Recreativos } \\
\text { de Base Natural } \\
\text { (RRBN) }\end{array}$ & $\begin{array}{l}\text { Puntaje promedio de } 7 \\
\text { variables }(\mathrm{A} 1)\end{array}$ & 10 & 20 \\
\hline & $\begin{array}{c}\text { Recursos Recreativos } \\
\text { Socialmente } \\
\text { Construidos } \\
\text { (RRSC) }\end{array}$ & $\begin{array}{l}\text { Puntaje promedio de } 4 \text { variables } \\
\text { (A2) }\end{array}$ & 10 & \\
\hline & $\begin{array}{c}\text { Problemas } \\
\text { Ambientales } \\
\text { (PA) }\end{array}$ & $\begin{array}{c}\text { Puntaje promedio de } 12 \\
\text { variables }(\mathrm{A} 3)\end{array}$ & 20 & 20 \\
\hline & \multicolumn{3}{|r|}{ Total } & 40 \\
\hline ICV & Total & & & 100 \\
\hline
\end{tabular}

\section{Dimensión socioeconómica de la calidad de vida}

"Se seleccionaron las variables intentando mostrar los tres aspectos fundamentales en su máxima y mínima expresión; basándonos en datos censales y estadísticas vitales disponibles para la unidad mínima (departamentos, comunas), privilegiando los aspectos estructurales y no la coyuntura inmediata (como epidemias o problemas circunstanciales).

Vivienda:

" Porcentaje de población en hogares hacinados, considerando como tales a aquellos que superan las dos personas por cuarto (Referencia $\mathrm{SE}_{1}$ ).

" Porcentaje de población que reside en hogares que carecen de inodoro de uso exclusivo o que tenga descarga de agua; nombrados como sin retrete (Referencia $\mathrm{SE}_{2}$ ).

» No existen datos confiables que permitan distinguir viviendas con alto grado de confort, por lo que sólo se incluyeron variables que reflejan requerimientos mínimos a satisfacer. 
Salud:

» Porcentaje de población sin cobertura por obra social, plan de salud privado o mutual. Esta variable también permite mostrar la existencia o no de precariedad laboral y discrimina mejor que la consideración de la sola presencia de hospitales, unidades sanitarias o recursos humanos destinados a la salud (Referencia $\mathrm{SE}_{3}$ ).

» Tasa de Mortalidad Infantil (TMI), o sea, el número de nacidos vivos y fallecidos antes de cumplir el año por cada mil habitantes de este grupo de edad, según lugar de residencia de la madre. Los datos se tomaron del Ministerio de Salud: Dirección de Estadísticas e Información de Salud (DEIS, 2013). Se considera como valor la media aritmética de los tres años peri-censales para disminuir las oscilaciones aleatorias propias de esta tasa. ${ }^{1}$ (Referencia $\mathrm{SE}_{4}$ ).

» Tampoco en este caso podemos medir acertadamente las máximas condiciones; pero sí inducimos las peores a partir de variables de carencia de los mínimos seleccionados para el índice. En ambos casos, la acción o inacción de los organismos públicos podría resolver en parte las deficiencias mostradas en lo que respecta a la salud.

\section{Educación:}

» Porcentaje de población de quince años o más que ya abandonó la escolarización y cuyo máximo nivel de instrucción alcanzado es menor a primario completo; como variable de mínima (Referencia $\mathrm{SE}_{5}$ ).

》 Porcentaje de población de quince años o más que ya alcanzó un nivel de instrucción universitario o postgrado completo; como variable de máxima (Referencia $\mathrm{SE}_{6}$ ).

\section{Dimensión ambiental de la calidad de vida}

Remitiéndonos a lo ya mencionado, a partir de 2010 es posible enriquecer el estudio de la calidad de vida incorporando también a los recursos recreativos, tanto los de base natural como los socialmente construidos, como variables pro-mejoras al bienestar, más allá de lo aportado por los problemas ambientales que son tomados como variables en detrimento de la calidad de vida.

Existen muchos menos precedentes con respecto a calidad ambiental, es decir, la incorporación de los recursos recreativos (y no la mera ausencia o escasa magnitud de los problemas ambientales) como variables que contribuyen al bienestar de la población. Por esta razón se creó toda una matriz de observación y un relevamiento exhaustivo respecto de la presencia, magnitud relativa y distancia de la población residente respecto de cada uno de los elementos seleccionados, para cada una de las unidades territoriales analizadas. Los detalles pueden ser consultados en un trabajo referido a la calidad ambiental en la Argentina (Velázquez y Celemín, 2013).

1 También hicimos pruebas con los quinquenios peri-censales (por ejemplo 1999-2002), pero el resultado terminaba ocultando algunas de las variaciones reales (Vega et al., 2006). 
Es importante aclarar que durante un quinquenio se consultó cada asignación de puntaje con investigadores locales y con visitas in situ de nuestros propios investigadores. La disponibilidad de páginas web municipales para todas las unidades funcionó como esqueleto estadístico y se fue enriqueciendo con otras fuentes que se mencionan para cada variable en el Cuadro 2.

Cuadro 2. Variables ambientales y sus fuentes de información.

\begin{tabular}{|c|c|}
\hline $\begin{array}{c}\text { RECURSOS RECREATIVOS } \\
\text { BASE NATURAL } \\
\text { (RRBN) }\left(\mathrm{A}_{1}\right) \\
\text { (Información Municipal/ terreno/ imágenes } \\
\text { satelitales) }\end{array}$ & $\begin{array}{c}\text { RECURSOS RECREATIVOS } \\
\text { SOCIALMENTE CONSTRUIDOS } \\
\text { (RRSC) }\left(\mathrm{A}_{2}\right) \\
\text { (Fuente: Información Municipal/ terreno). }\end{array}$ \\
\hline $\begin{array}{l}\text { Presencia, calidad y accesibilidad popular } \\
\text { de: } \\
\text { 1-Playas. } \\
\text { 2- Balnearios a orillas de ríos, lagos, lagunas } \\
\text { o diques. } \\
\text { 3-Centros termales. } \\
\text { 4-Nieve/hielo (posibilidad de actividades } \\
\text { recreativas). } \\
\text { 5-Relieve (paisaje). } \\
\text { 6-Espejos y cursos de agua (paisaje). } \\
\text { 7-Parques y espacios verdes (esparcimiento). }\end{array}$ & $\begin{array}{l}\text { Presencia, calidad y accesibilidad } \\
\text { popular de: } \\
\text { 1-Estética/Patrimonio urbano. } \\
\text { 2-Centros culturales. } \\
\text { 3-Centros comerciales y de esparcimiento. } \\
\text { 4-Centros deportivos. }\end{array}$ \\
\hline
\end{tabular}

PROBLEMAS AMBIENTALES (PA) $\left(\mathrm{A}_{3}\right)$

1-Uso de plaguicidas en agricultura.

(Defensoría del Pueblo. Atlas Ambiental de la Niñez, 2009).

2-Participación de Industria y minería en el PBG.

(INDEC, 2003).

3-Contaminación/ Ruido/Congestionamiento (Información Municipal/terreno/escala urbana)

4- Localizaciones peligrosas.

(Información Municipal/terreno/imágenes satelitales).

5-Localizaciones con Externalidades negativas.

(Información Municipal/terreno/imágenes satelitales).

6-Inseguridad. Tasa de hechos delictivos por cada 10.000 habitantes.

(Dirección Nacional de Política Criminal, 2008).
7-Asentamientos precarios. \% de población residente en villas miseria.

(Argentina. INDEC. Censo Nacional 2001, Información inédita, 2004).

8-Basurales. \% de población residente a menos de 300 metros de un basural a cielo abierto.

(Argentina. INDEC. Censo Nacional 2001, Información Inédita, 2004).

9-Sismicidad y vulcanismo.

(Chiozza et al, 1987).

10-Tornados.

(Geosistemas, 1997).

11-Inundabilidad.

(Argentina. INDEC. Censo Nacional 2001, Información inédita, 2004).

12-Malestar climático.

(IRAM, 1996).

\section{Comparación general de indicadores de calidad de vida para 1991-2010}

Como puede observarse en el Cuadro 3, las estadísticas censales y vitales consideradas en el $\mathrm{ICV}^{2}$ permiten afirmar que las condiciones socioeconómicas de la población

2 No efectuamos comparaciones respecto de las variables ambientales, ya que las utilizadas a partir del 2010 son más (23 en total) y más complejas (12 sobre problemas ambientales, 7 sobre RRBN y 4 sobre RRSC) que las utilizadas precedentemente (inundabilidad, sismicidad, tornados, erosión de suelos y viviendas de fin de semana). 
argentina mejoraron considerablemente durante el último período inter-censal. El complicado panorama del 2001 se revirtió en gran medida para el 2010. A continuación mostraremos el cálculo de diferentes indicadores significativos, considerando los individuos/hogares argentinos promedio relevados en cada ocasión.

Cuadro 3. Evolución de indicadores socio-económicos en Argentina 1991-2010. Fuente: Censos Nacionales (1991, 2001 y 2010), DEIS (2013), Ministerio de Salud y elaboración del autor.

\begin{tabular}{c|c|c|c|c|c|c}
\hline \multirow{2}{*}{ Años } & \multicolumn{2}{|c|}{ Vivienda } & \multicolumn{2}{c|}{ Salud } & \multicolumn{2}{c}{ Educación } \\
\cline { 2 - 7 } & $\begin{array}{c}\text { (SE1) } \\
\text { Sin retrete } \\
\%\end{array}$ & $\begin{array}{c}(\text { SE2) } \\
\text { Hacinados } \\
\%\end{array}$ & $\begin{array}{c}(\text { SE3) } \\
\text { TMl } \%\end{array}$ & $\begin{array}{c}\text { Sin obra } \\
\text { social }\end{array}$ & $\begin{array}{c}\text { (SE5) } \\
\text { Educación } \\
\text { menor a } \\
\text { Primaria } \%\end{array}$ & $\begin{array}{c}\text { (SE6) } \\
\text { Educación } \\
\text { Universitaria } \\
\%\end{array}$ \\
\hline 1991 & 21,86 & $27,20\left(^{*}\right)$ & 21,70 & 37,76 & 22,08 & 3,81 \\
\hline 2001 & 16,86 & 29,15 & 16,60 & 48,05 & 17,90 & 4,39 \\
\hline 2010 & $15,16\left(^{* *}\right)$ & 28,06 & 11,90 & 36,08 & 12,86 & 6,84 \\
\hline
\end{tabular}

(*) El dato corresponde a porcentaje de hogares.

$\left.{ }^{(\star \star}\right)$ El dato corresponde a población sin retrete con descarga de agua.

Nota: la tabla completa con los valores de cada unidad se encuentra en Velázquez (2001, 2008 y 2016).

Cómo los mapas pueden mostrar más que lo dicho en muchas palabras, a continuación de cada descripción del promedio, ofrecemos una selección del ICV a nivel departamental/comunal (525 unidades). Siempre tomando al año 2010 como base (mejor situación relativa o, en otros términos, logro mayor); por eso los intervalos de los mapas de 2001 y 2010 son los mismos.

Los indicadores de hacinamiento son los que muestran menos avances. Al deterioro de las viviendas existentes se suma la aún insuficiente construcción de nuevas unidades destinadas a sectores populares. El segmento más dinámico en cuanto a construcción siguió constituyéndolo la demanda solvente en búsqueda de oportunidades de inversión más que con intención de residir en las nuevas viviendas (Cisterna y Matteucci, 2015). A partir del censo de 2010 se implementaron políticas públicas que directamente buscaron mejorar el acceso a la vivienda única propia a partir de líneas de crédito subsidiadas por los bancos y planes directos de construcción, entre ellos el PROCREAR. En cuanto a la presencia de retrete, se observan mejoras desde 1991 en forma continua. 

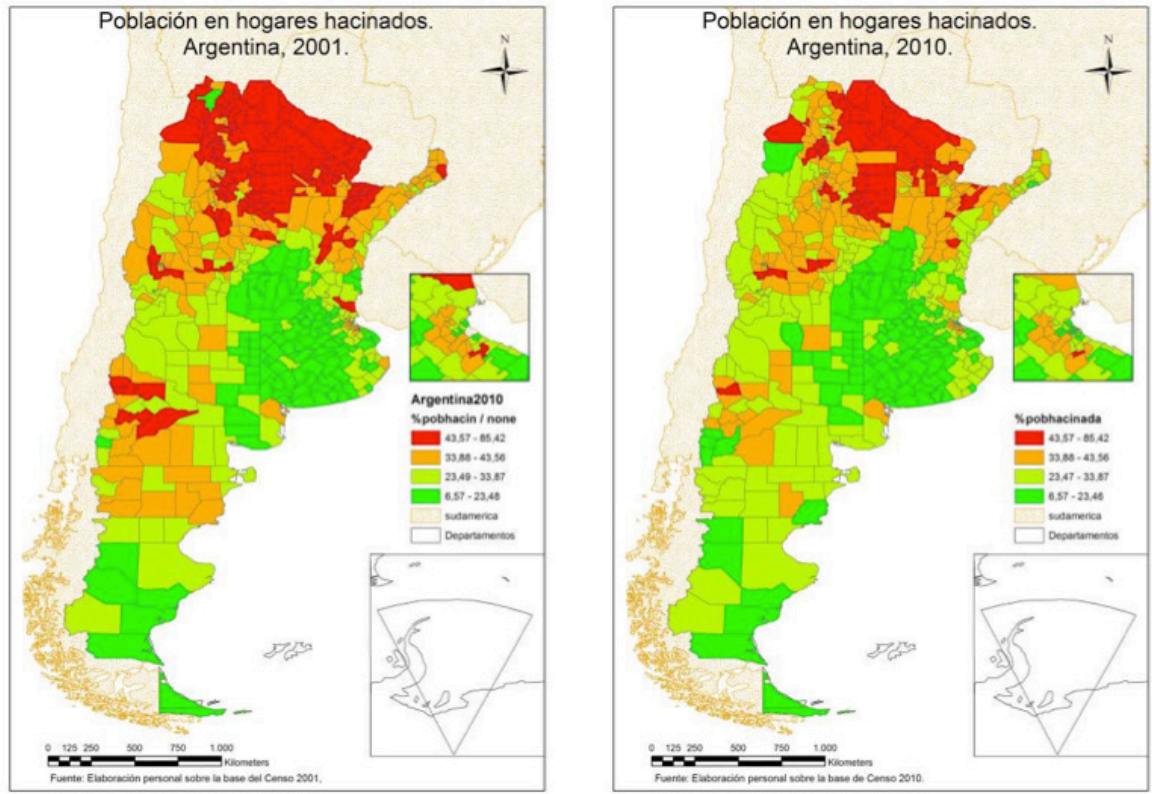

Figura 1. Indicador de vivienda: hacinamiento en 2001 y 2010. Fuente: elaboración del autor con REDATAM, sobre datos censales de 2001 y 2010.
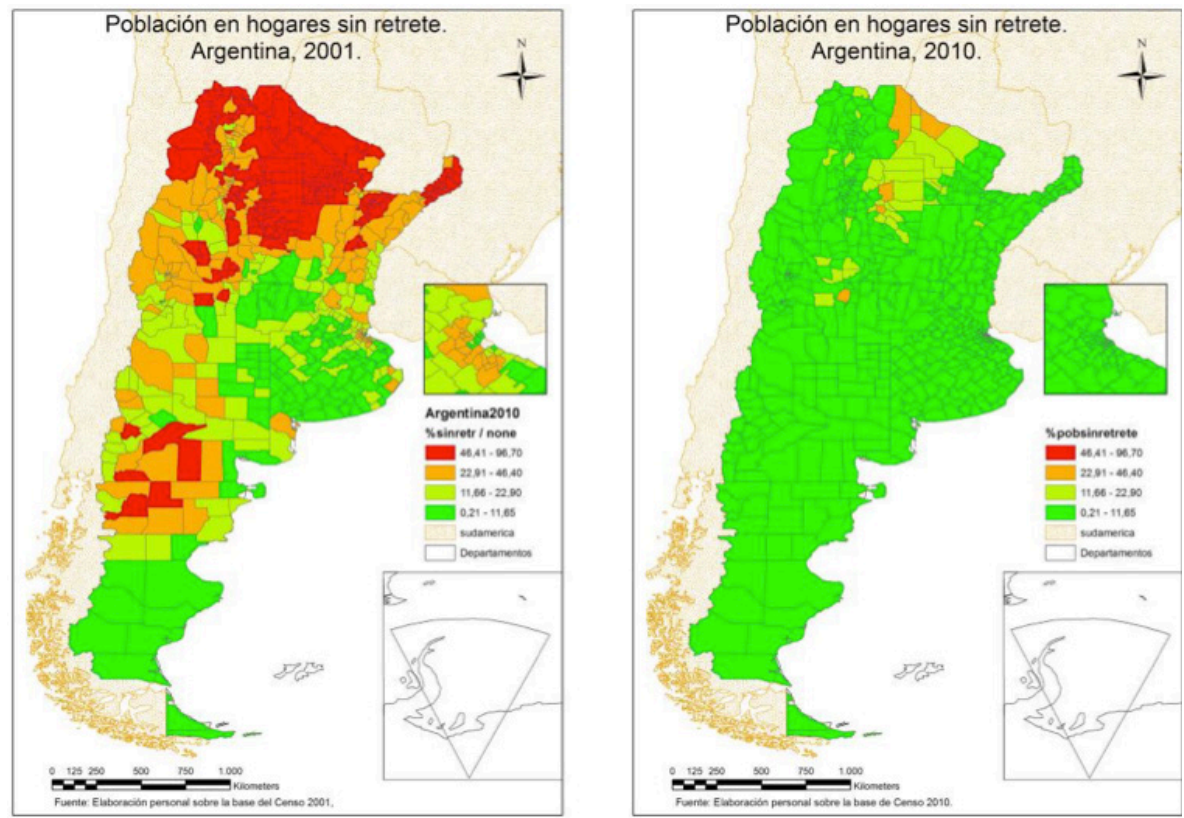

Figura 2. Indicador de vivienda: Sin retrete en 2001 y 2010. Fuente: elaboración del autor con REDATAM, sobre datos censales de 2001 y 2010.

Los indicadores de salud entre 1991 y 2010 muestran una disminución de la mortalidad infantil en términos generales y, como veremos, en forma sustantiva en algunas de las jurisdicciones más vulnerables; todo esto más allá de lo considerado como razonable en virtud del proceso de transición demográfica. Esta mejora se explicaría, en parte, por el incremento del presupuesto de salud (Van der Kooy y Pezzella, 2013) también, por la mejora en la formalidad y retribución de los trabajadores (Bertranou y Casanova, 2014) 
Geografía y cambios en la calidad...

GUILLERMo ANGel VeLÁzquez

y además, fundamentalmente entre el 2001 y el 2010, por la inclusión en el régimen de jubilación de trabajadores que habrían quedado excluidos en períodos anteriores (Basualdo, 2009). Cabe observar, especialmente, el pico de población sin cobertura de obra social que se registra durante la crisis del 2001.
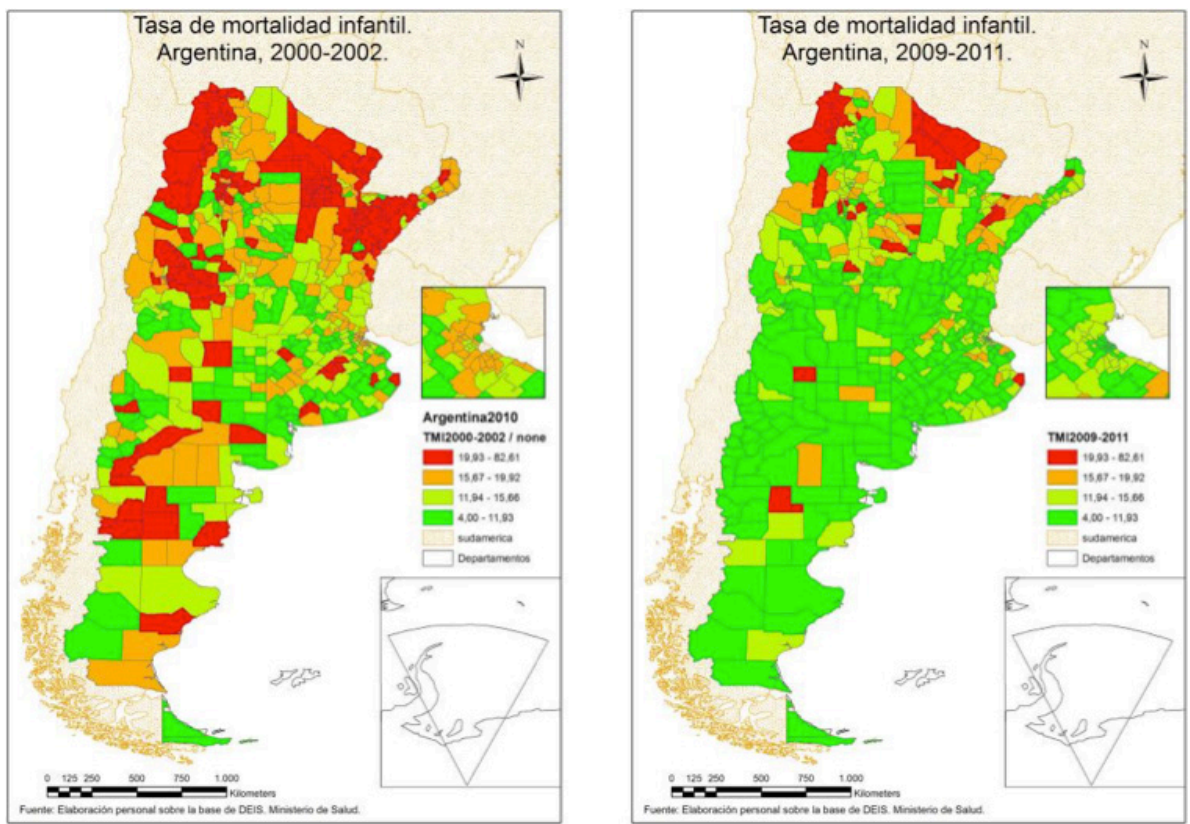

Figura 3. Indicador de salud: TMI en años peri-censales 2001 y 2010. Fuente: elaboración personal sobre datos Dirección de Estadística e Información de Salud y REDATAM y sobre datos censales de 2001 y 2010.
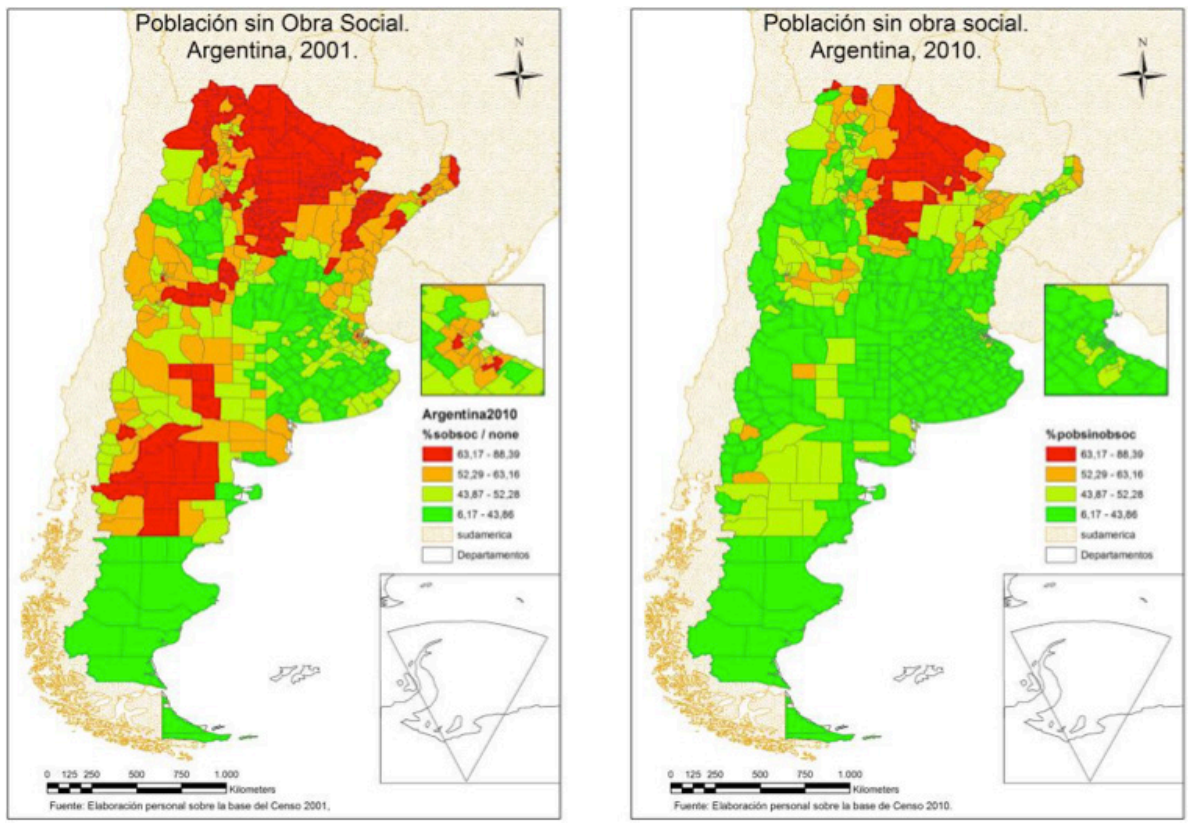

Figura 4. Indicador de salud: Sin Obra Social 2001 y 2010. Fuente: elaboración del autor sobre REDATAM y datos censales de 2001 y 2010. 
Geografía y cambios en la calidad...

GUILLERMo ANGel VeLÁzquez

Las políticas públicas tuvieron efecto positivo para mejorar la finalización de la escolaridad primaria; puede observarse el mismo progreso desde 1991. Esto se vincula con el claro objetivo de contener a los jóvenes dentro del sistema educativo formal, aunque probablemente en un contexto de persistencia de la fragmentación de los circuitos educativos. A partir del 2003, de la mano del incremento presupuestario a la educación superior, se crearon varias universidades en diferentes puntos del país, aumentando así la oportunidad de acceso a títulos universitarios de sectores de la población otrora excluidos.
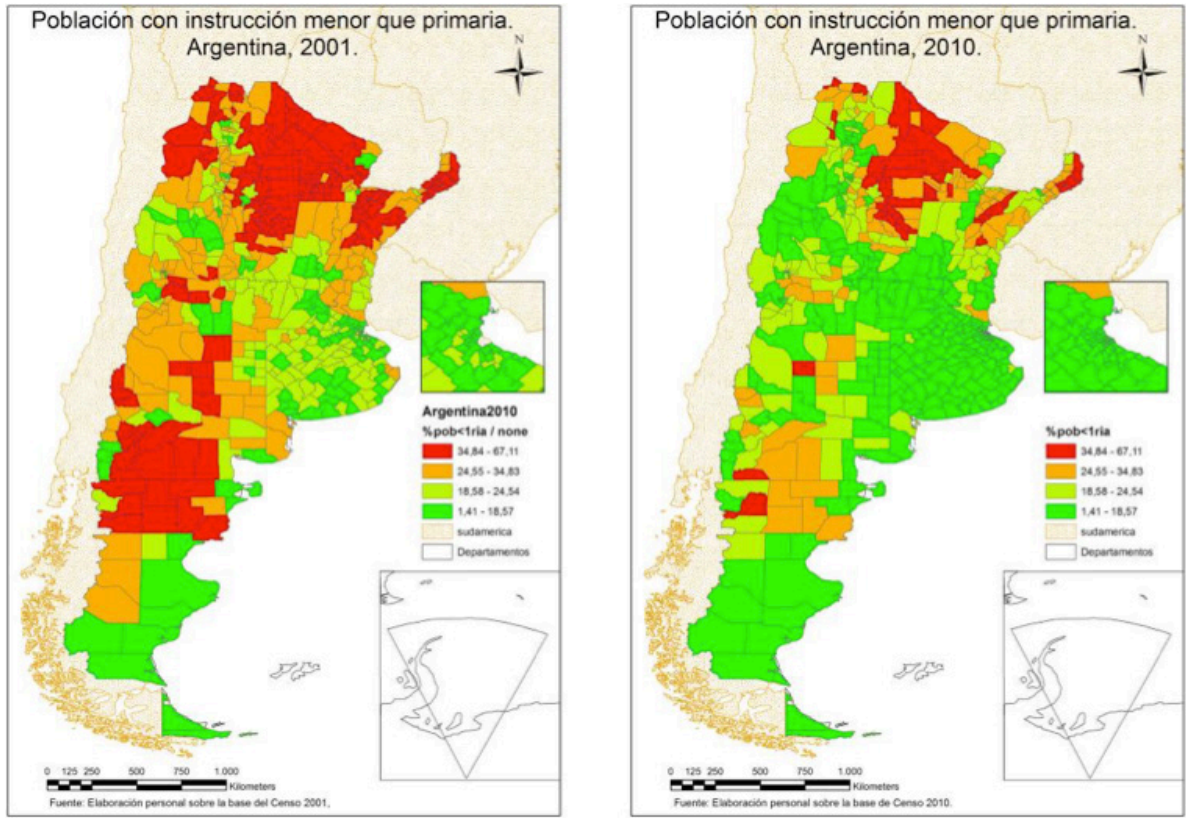

Figura 5. Indicador de educación: menor a primaria 2001 y 2010. Fuente: elaboración del autor REDATAM y sobre datos censales de 2001 y 2010.
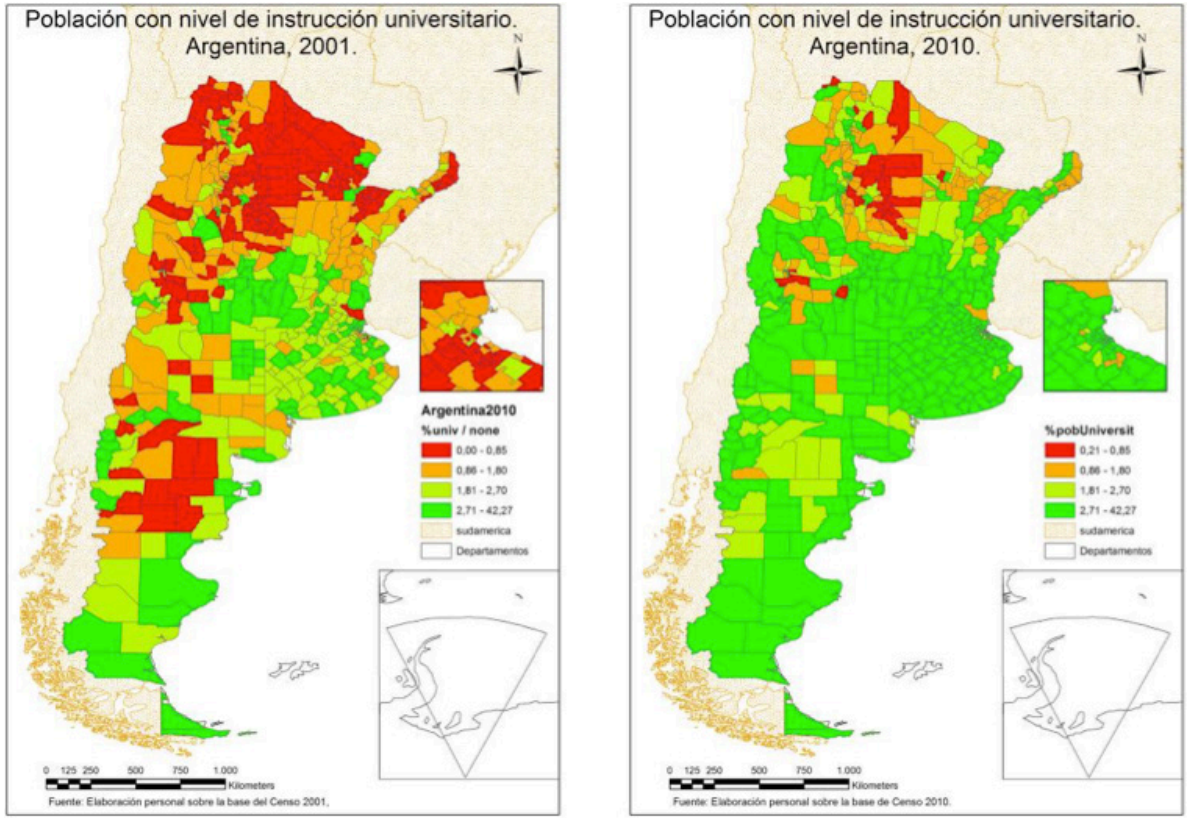

Figura 6. Indicador de educación: Universitarios en 2001 y 2010. Fuente: elaboración del autor con REDATAM, sobre datos censales de 2001 y 2010. 


\section{Comparación general en términos de número índice de calidad socioeconómica 1991-2010}

Para poder dimensionar la importancia del cambio registrado durante este último lapso, debemos considerar los valores del Cuadro 2, pero referirlos al máximo y al mínimo del Censo base, que es el último de 2010. Dicho de otro modo, estudiamos la posición relativa de la variable, por ejemplo TMI con valores de 1991, pero con la posición relativa que este valor hubiera obtenido con los estándares del 2010.

El Cuadro 4 muestra este ajuste para todas las variables socioeconómicas de 1991, 2001 y 2010 con respecto la última medición considerada como base. ${ }^{3}$

Cuadro 4. Índices promedio de indicadores socio-económicos en Argentina.1991-2010. Fuente: ajuste matemático del autor con índices omega a partir los valores del Cuadro 3, según los siguientes máximos y mínimos del 2010.

\begin{tabular}{|c|c|c|c|c|c|c|c|c|c|c|c|}
\hline \multirow[b]{2}{*}{ Años } & \multicolumn{4}{|c|}{ Vivienda } & \multicolumn{3}{|c|}{ Salud } & \multicolumn{4}{|c|}{ Educación } \\
\hline & \multicolumn{2}{|c|}{$\begin{array}{l}\left(S_{1}\right) \operatorname{Sin} \\
\text { retrete \% }\end{array}$} & \multicolumn{2}{|c|}{$\begin{array}{c}\left(\mathrm{SE}_{2}\right) \\
\text { Hacinados } \\
\%\end{array}$} & \multicolumn{2}{|c|}{$\begin{array}{c}\left(\mathrm{SE}_{3}\right) \mathrm{TMI} \\
\%\end{array}$} & $\begin{array}{c}\left(\mathrm{SE}_{4}\right) \operatorname{Sin} \\
\text { obra social } \\
\%\end{array}$ & \multicolumn{2}{|c|}{$\begin{array}{c}\left(\mathrm{SE}_{5}\right) \text { Educación } \\
\text { menor a Primaria } \\
\%\end{array}$} & \multicolumn{2}{|c|}{$\begin{array}{c}\left(\mathrm{SE}_{6}\right) \\
\text { Educación } \\
\text { Universitaria \% }\end{array}$} \\
\hline 1991 & \multicolumn{2}{|l|}{0,49} & \multicolumn{2}{|c|}{0,73} & \multicolumn{2}{|c|}{0,51} & 0,56 & \multicolumn{2}{|l|}{0,56} & & 0,09 \\
\hline 2001 & \multicolumn{2}{|c|}{0,51} & \multicolumn{2}{|c|}{0,71} & \multicolumn{2}{|c|}{0,65} & 0,42 & \multicolumn{2}{|l|}{0,65} & & 0,10 \\
\hline 2010 & \multicolumn{2}{|l|}{0,65} & 0 & & \multicolumn{2}{|c|}{0,78} & 0,59 & \multicolumn{2}{|l|}{0,76} & & 0,16 \\
\hline \multirow{3}{*}{\multicolumn{2}{|c|}{ Valores 2010}} & & ximo & & & 84,3 & 40,00 & 78,74 & 48 & & 42,27 \\
\hline & & & nimo & & & 6,57 & 4,00 & 6,17 & 1,4 & & 0,21 \\
\hline & & & & & & “a” & "a" & "a" & "a & & "b" \\
\hline
\end{tabular}

\section{Cálculo explicativo de referencia}

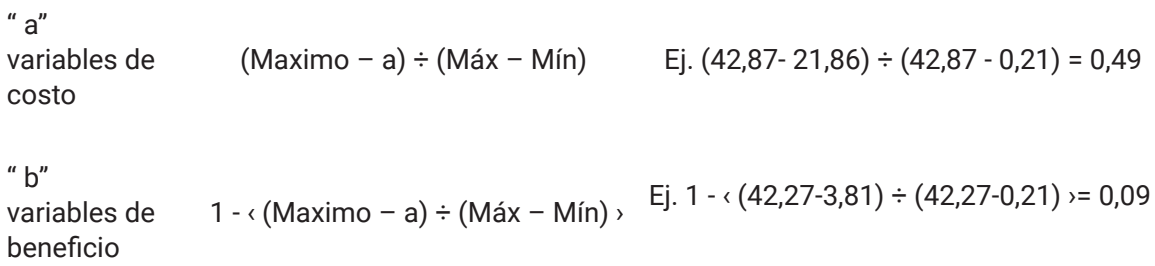

A partir de los números-índice del cuadro 4 resulta posible establecer los índices de calidad de vida "promedio" para cada año; recordemos que las variables referidas a calidad ambiental, que tan útiles resultan para el análisis de cada año en particular, no son consideradas debido a la modificación para 2010 (su peso relativo se acrecienta de un $20 \%$ a un $40 \%$ y como ya explicamos, hay un enriquecimiento en sus componentes); en otras palabras, le asignamos el mismo índice ambiental para todos los habitantes, por lo que su efecto en los cálculos es neutro.

3 Para transformar en números índice (base 2010) las tasas de 1991 y 2001, se tomaron las tasas de cada año y se las refirió al máximo y mínimo del 2010. Por ejemplo: la TMI de 1991 (21,7 por mil), referida a los mínimos y máximos departamentales de TMI del 2010 (4 y 40 por mil respectivamente) se corresponde con un índice de 0,51. En todos los casos un índice mayor indica mejor situación relativa (tanto para las variables de costo como para las de beneficio). Recordemos que las variables ambientales utilizadas no son las mismas, por lo que no pueden ser estandarizadas para los tres años. 
GUILLERMo ANGEL VeLÁZqueZ

Cuadro 5. Análisis de la evolución de los índices del Cuadro 4, 1991-2010. Fuente: elaboración del autor a partir del Cuadro 4.

\begin{tabular}{l|c|c|c|c}
\hline Año & $\begin{array}{c}\text { ICV } \\
(\text { Max 10 y Min 1) } \\
(\star \star \star\end{array}$ & $\begin{array}{c}\text { Incremento } \\
\text { porcentual } \\
\text { inter-censal }\end{array}$ & $\begin{array}{c}\text { Longitud del } \\
\text { período }\end{array}$ & $\begin{array}{c}\text { Tasa anualizada del } \\
\text { incremento }\end{array}$ \\
\hline 1991 & 4,91 & & & \\
\hline 2001 & 5,23 & $6,60 \%$ & 10,5 años & $0,63 \%$ \\
\hline 2010 & 6,09 & $16,39 \%$ & 9 años & $1,82 \%$ \\
\hline
\end{tabular}

$(\star \star \star)$ Se promedian las variables socioeconómicas (todas pesan igual, Cuadro 1) y luego se ajusta el peso relativo obtenido entre 0 y 1 , a valores de escala 1 a 10, que es la reflejada en el ICV de los mapas expuestos. Se computaron los valores originales con todos sus decimales y no los redondeos a dos cifras.

Vemos claramente que entre 1991 y 2001 el ICV no creció siquiera 1 punto interanual, mientras que entre 2001 y 2010 creció en casi 2 puntos por año; siempre hablando de promedios.

El modesto incremento de la calidad de vida de1991 a 2001 (o,63\% promedio anual) se debió más a las características de inercia propias de la transición demográfica que a mejoras reales durante la década de los noventa. Usando los valores del Cuadro 3, podemos explicar que, según la teoría de la transición demográfica, una paulatina mejora en la mortalidad infantil era algo previsible (o,51 contra o,65 de un máximo de 1), pero no se esperaba que, tal como ocurrió, la cobertura médico-social disminuyera drásticamente asociándose esto con el incremento del desempleo y de la precarización laboral (cae de 0,56 a 0,42, respecto de un máximo de 1). Cabe destacar que de no haberse incluido esta variable entre las de salud, el daño generado durante los noventa hubiera pasado desapercibido. También parecía natural esperar avances en cuanto a presencia de retrete en los hogares (0,49 contra 0,51 respecto de un máximo de 1); pero es marcado el retroceso en vivienda debido a un empeoramiento en la variable hacinamiento (de o,73 cae a 0,71 respecto de un máximo de 1). Los avances en escolarización fueron también modestos (primaria completa trepó de o,56 a o,65 y universitarios lo hizo de 0,09 a 0,10, siempre respecto de un máximo de 1).

Por lo expuesto, los escasos incrementos en las variables de calidad de vida que retenemos muestran un desempeño muy débil de las políticas públicas de esos años que se caracterizaron por un programa de gobierno neoliberal.

Durante la última década (2001-2010) la situación de la calidad de vida de grandes segmentos de la población argentina es completamente diferente. La tasa de crecimiento del índice es holgada, alcanzado el 1,82\% de promedio anual; o sea, se triplicó respecto del período anterior. También se registraron avances sustanciales en educación y salud atribuibles a políticas públicas activas durante estos años. ${ }^{4}$ Aunque por otro lado, como señalamos, en lo que respecta a vivienda los logros registrados hasta octubre del 2010 resultaron aún muy modestos.

Estos elocuentes números generales permiten afirmar que la primera década de nuestro siglo conoció un indiscutible mejoramiento en la calidad de vida de la población que, sin

4 En este punto hay cuestionamientos de algunos sectores respecto de la validez de los datos suministrados por el Sistema Estadístico Nacional. Sin embargo, estos logros han sido reconocidos por diversos organismos internacionales como la UNICEF, OEA, Banco Mundial, FMI, etc. 
duda, puede ser atribuido al giro de las políticas públicas que, luego del fracaso de las gestiones anteriores que terminó con la gran crisis de 2001, optó por una orientación diametralmente opuesta.

\section{Índice de calidad de vida: su análisis por departamentos y regiones. Comparación 2001-2010 (mismos intervalos)}
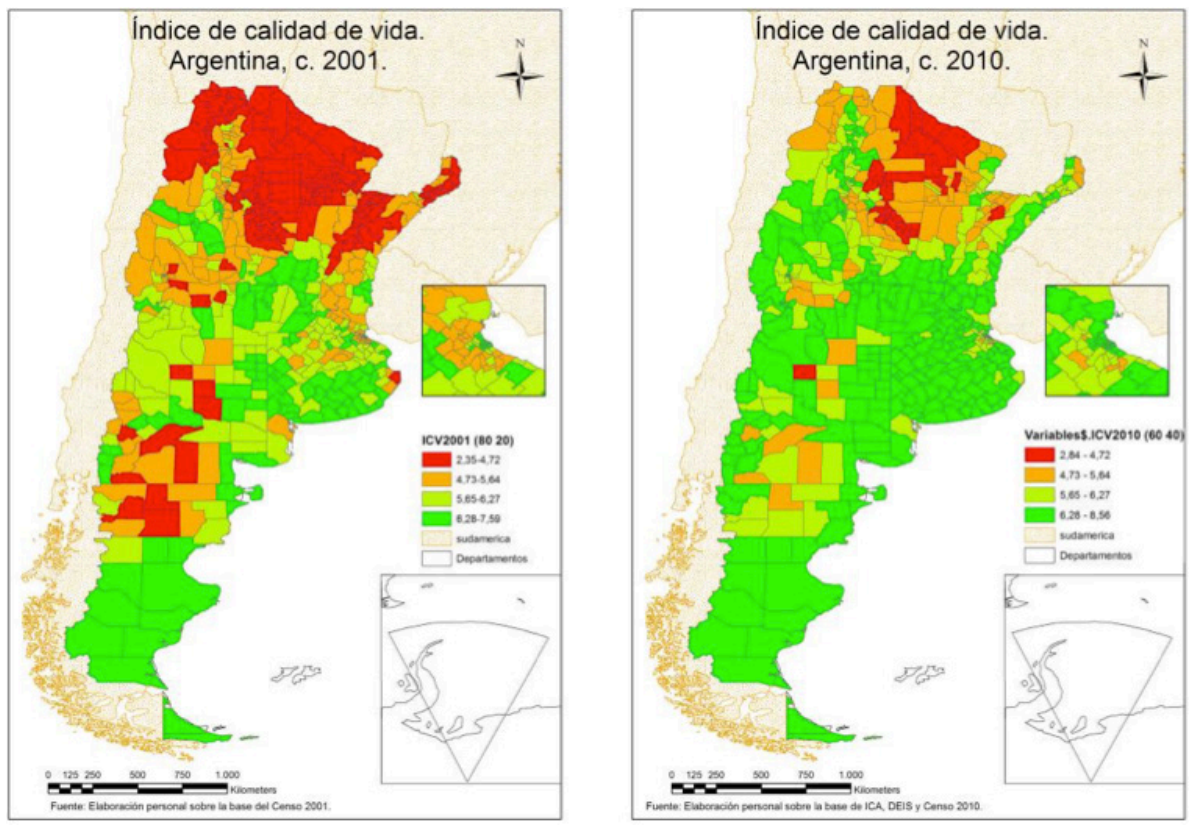

Figura 7. Índice de Calidad de Vida para 2001 y 2010. Fuente: elaboración del autor sobre la base de ICA (Velázquez, Celemín, 2013), Dirección de Estadística e Información de Salud y Censos 2001 y 2010, con REDATAM, sobre censos 2001 y 2010,

Resulta elocuente la disminución del área y, por consiguiente, de la población afectada por bajas condiciones de vida entre 2001 y 2010. Esta situación queda circunscripta casi exclusivamente al norte, más particularmente a la cuña más aislada y despoblada que se interpone entre el Noroeste Argentino (NOA) y el Nordeste Argentino (NEA). También persisten algunos relictos en el corazón de Corrientes. Fuera del norte, el único departamento que aún persiste en esta situación es Chical Có en La Pampa.

Por otra parte, el área y población con mejores condiciones de vida avanzó muchísimo en todo el norte entre 2001 y 2010, abarcando a las áreas más urbanizadas y accesibles y alcanzado sectores de provincias que desconocían por completo esta situación en el 2001 (Formosa, Chaco, Corrientes). En las restantes regiones esta situación de mayor bienestar relativo se extiende más y abarca también a mucha más población. Es casi la pauta general en Cuyo (fundamentalmente en las áreas de oasis más poblados). Lo mismo ocurre en la Región Pampeana, salvo en sus periferias norte y oeste y algunos sectores de la pampa deprimida. Respecto de la Región Metropolitana de Buenos Aires (RMBA), es la situación predominante en las áreas más consolidadas de CABA y del 
resto de la conurbación. El resto se sitúa en segunda y tercera posición. ${ }^{5}$ Finalmente, en la Patagonia la situación ampliamente mayoritaria es de alta calidad de vida. Sólo en algunas áreas de la meseta se desciende al segundo y tercer nivel de la escala.

Cuadro 6. Calidad de vida en 2001 y 2010. Índice, cantidad de departamentos y población involucrada. Fuente: elaboración del autor sobre la base de base de datos de ICV 2001 e ICV 2010.

\begin{tabular}{l|c|c|c|c}
\hline ICV & \multicolumn{2}{|c|}{2001} & \multicolumn{2}{c}{2010} \\
\hline & Población (miles) & $N^{\circ}$ departamentos & Población (miles) & $\begin{array}{c}N^{\circ} \text { departamentos } \\
\text { / comunas }\end{array}$ \\
\hline $\begin{array}{l}\text { muy bajo } \\
(2,35-4,72)\end{array}$ & $3.329,4$ & 132 & 536,5 & 27 \\
\hline $\begin{array}{l}\text { bajo } \\
(4,73-5,64)\end{array}$ & $10.870,4$ & 132 & $2.661,0$ & 76 \\
\hline $\begin{array}{l}\text { medio } \\
(5,65-6,27)\end{array}$ & $11.934,3$ & 129 & $8.514,5$ & 117 \\
\hline $\begin{array}{l}\text { alto } \\
(6,28-8,56)\end{array}$ & $10.087,9$ & 117 & $28.324,3$ & 305 \\
\hline
\end{tabular}

En el año 2001 había 3,3 millones de argentinos que residían en el 25\% de los departamentos caracterizados por muy bajos índices de calidad de vida. En el otro extremo, 10,1 millones residían en el 25\% de departamentos con las mejores condiciones. La situación en el 2010 mejora ostensiblemente: el grupo con peor situación se reduce a tan sólo 27 unidades en donde residen poco más de medio millón de habitantes. Cabe destacar que lograr disminuir de más de 3 millones a poco más de medio millón a los argentinos que padecen muy malas condiciones de vida es un hecho impactante $\mathrm{e}$ inédito, que nunca habíamos registrado desde que estudiamos la calidad de vida de la población argentina a escala departamental (década del ochenta).

En la situación opuesta, el grupo de población en departamentos con alta calidad de vida aumentó en el 2010 a 28,3 millones de personas que residen en 305 unidades. Esto implica haber multiplicado casi por tres a los argentinos que pueden gozar de mejores condiciones de vida durante la primera década del XXI.

Cuadro 7. Índice de Bienestar y posición por regiones, 2001 y 2010. Fuente: elaboración del autor a partir de los mapas de ICV 2001 y 2010.

\begin{tabular}{l|c|c|c}
\hline Región & & 2001 & 2010 \\
\hline Patagonia & $1^{\circ}$ & 6,48 & 7,03 \\
\hline Pampeana & $2^{\circ}$ & 6,18 & 6,93 \\
\hline Cuyo & $3^{\circ}$ & 6,05 & 6,91 \\
\hline Metropolitana & $4^{\circ}$ & 5,94 & 6,77 \\
\hline NOA & $5^{\circ}$ & 5,35 & 6,33 \\
\hline NEA & $6^{\circ}$ & 4,62 & 6,01 \\
\hline
\end{tabular}

5 En el caso de la RMBA la escala de partidos (incluso de comunas) puede resultar insuficiente para captar la magnitud de las diferencias. Recomendamos, al respecto, el trabajo de Manzano et al. 2016, que efectúa este análisis a escala de fracción censal. 


\begin{tabular}{l|c|c}
\hline sigma & 0,67 & 0,40 \\
\hline media & 5,77 & 6,66 \\
\hline mediana & 6,00 & 6,84 \\
\hline Coeficiente de Variación & $11,67 \%$ & $6,06 \%$ \\
\hline
\end{tabular}

Vemos que la Patagonia es, en términos generales, la región con mayor calidad de vida en la Argentina. Por el contrario, el Nordeste continúa siendo la más deficitaria en este aspecto, seguida por el Noroeste. Las Regiones Pampeana y Cuyana se sitúan claramente por arriba del promedio general, mientras que la Región Metropolitana lo había estado hasta 1991, pero luego no logra sostener esta situación relativa, fundamentalmente en relación con el deterioro de su calidad ambiental y el mayor avance relativo en los aspectos socioeconómicos de las regiones Pampeana y Cuyana, que logran superarla.

\section{Pronósticos y puntos destacables}

Más allá de la estabilidad en las posiciones regionales, se destaca por su relevancia la tendencia a la convergencia que se observa entre 2001 y 2010. La distancia relativa entre las regiones peor y mejor posicionadas se reduce casi a la mitad (casi 2 puntos en 2001 contra 1 en el año 2010). El achicamiento del coeficiente de variación a casi la mitad también muestra una disminución de la brecha entre las diferentes regiones argentinas. Si bien persisten diferencias regionales, su grado se ha reducido considerablemente.

Consideramos que el cambio en las políticas públicas, implementado a partir de diciembre del 2015 por la nueva administración nacional, probablemente impactará negativamente en esta convergencia, que podría considerarse como mayor equidad territorial relativa de la calidad de vida, en la medida en que se vuelvan a adoptar orientaciones comparables a las de la década de 1990.

En las regiones empobrecidas (Nordeste y Noroeste) muy probablemente se agudizarán los problemas existentes en virtud del achicamiento del mercado doméstico al cual están destinados la mayoría de sus productos. Sólo una pequeña elite, con potencial exportador, podrá sustraerse de esta situación.

La Patagonia es, como dijimos, una región cuya posición relativa se explica, en cierta medida, por la intervención del Estado, por lo que, muy probablemente, empeore su situación relativa. Ya hay fuertes indicios de emigración de retorno en Tierra del Fuego por el cierre de plantas industriales y la disminución del flujo turístico doméstico, mientras que en Santa Cruz la administración provincial se encuentra en crisis.

En la Región Metropolitana, corazón industrial y de servicios, muy probablemente se agudizarán las fragmentaciones sociales y las conflictividades preexistentes que tienen allí su epicentro.

En la Región Pampeana los sectores vinculados con la exportación agropecuaria, fundamentalmente los grandes agentes, serán los grandes ganadores de la desregulación y apertura comercial. El resto deberá pujar por mantener su situación relativa. 
Por último, en la Región Cuyana los sectores dirigidos a la exportación (algunos segmentos de la vitivinicultura y la megaminería) probablemente puedan alcanzar mejoras relativas; por el contrario, los sectores vinculados con productos dirigidos al mercado interno, muy probablemente, experimenten retrocesos.

En síntesis: ya hay claros indicios de retroceso de los sectores asalariados en favor de los empresariales en virtud del incremento de la desocupación, el deterioro del salario real o las reformas impositivas que arrojan una estructura más regresiva de la distribución de la riqueza. En este sentido, la magnitud de las diferencias en la sociedad y el territorio argentino muy probablemente vuelvan a incrementarse en caso de persistir el cambio iniciado en diciembre de 2015. 


\section{Q Bibliografía}

»Defensoría del Pueblo de La Nación (2009). Atlas del Riesgo ambiental de la niñez de la Argentina. Buenos Aires: PNUD-UNICEF-OPS-OIT. Recuperado de http:// defensoresymedios.org.ar/wp-content/uploads/2010/04/Atlas.pdf

»DEIS. (2013). Buenos Aires: Dirección de Estadística e Información de Salud. Datos inéditos solicitados a informacion@deis.gov.ar.

» Dirección Nacional de Política Criminal. (2008). Estadísticas en Materia de Criminalidad. Ministerio de Justicia, Seguridad y Derechos Humanos. Presidencia de la Nación. Recuperado de http://www.jus.gov.ar/areas-tematicas/estadisticasen-materia-de-criminalidad.aspx

» INDEC (1994). Censo Nacional de Población, Hogares y Vivienda 1991. Buenos Aires: Instituto Nacional de Estadísticas y Censos.

» INDEC (2003). PBG por provincia y sector de actividad económica. Recuperado de http://www.mecon.gov.ar/secpro/dir_cn/documentos/producto_bruto_geografico. xls

» INDEC (2004). Censo Nacional de Población, Hogares y Viviendas 2001. Recuperado de www.indec.mecon.gov.ar.

» INDEC (2004). Censo nacional de población, hogares y vivienda 2001. Buenos Aires, 2004. Trabajo especial de procesamiento de variables de hábitat 22.144/04. Buenos Aires: Instituto Nacional de Estadísticas y Censos.

" INDEC (2013). Censo Nacional 2010 de población, hogares y viviendas. Resultados definitivos, por provincias, departamentos y localidades. Base de datos REDATAM. Buenos Aires: Instituto Nacional de Estadísticas y Censos.

» INDEC (2014). Argentina 2014. Buenos Aires: Instituto Nacional de Estadísticas y Censos.

》IRAM (1996). Clasificación bioambiental de la República Argentina. Buenos Aires: Instituto Argentino de Normalización.

» Barclay, G. (1962). Técnicas de Análisis de la Población. Rosario: Instituto Interamericano de Estadística. Comisión de Educación.

» Basualdo, E. (2009). La evolución del sistema previsional argentino. Buenos Aires: CIFRA.

》 Bertranou, F. y Casanova, L. (2014). Informalidad laboral en Argentina. Buenos Aires: OIT.

» Bolsi, A., Longhi, F. y Paolasso, P. (2006). El Norte Grande Argentino entre el progreso y la pobreza. Población \& Sociedad, 12-13, pp. 231-270

»Celemín, J. P. (2007). El estudio de la calidad de vida ambiental: definiciones conceptuales, elaboración de índices y su aplicación en la ciudad de Mar del Plata, Argentina. Hologramática, 1(7), 71-98.

» Cisterna, C. y Matteucci, L. (2015). La urbanización en argentina durante el neodesarrollismo. Una lectura desde las rupturas y continuidades con el período neoliberal. Revista de Direito da Cidade, 7(4), 1573-1599. 
» Chiozza, E. y Figueira, R. (1981-1987). Atlas Total de la República Argentina. Buenos Aires: Centro Editor de América Latina.

»Connerly, C. y Marans, R. (1985). Comparing global measures of perceived neighborhood quality. Social Indicators Research, 17, 29-47.

» Delgado, M. T. y Méndez, E (1996). Planificación territorial. Medio ambiente y calidad de vida. Mérida: Litorama.

"Estés, R. (1993). Hacia un índice de calidad de vida: enfoques empíricos para la evaluación del bienestar humano a nivel internacional. En B. Kliksberg, Pobreza, un tema impostergable (pp. 132-148). Caracas: FCE-PNUD, UNESCO.

»Fernández-López, J. A., Fernández-Fidalgo, M. y Cieza, A. (2010). Los conceptos de la calidad de vida, salud y bienestar analizados desde la perspectiva de la clasificación internacional del funcionamiento (CIF). Revista Española de Salud Pública, 84(2),169-84.

» Friel, S., Akerman, M., Hancock, T., Kumaresan, J., Marmot, M., Melin, T. y Vlahov, D. (2011). Addressing the social and environmental determinants of urban health equity: evidence for action and a research agenda. Journal of Urban Health, 88(5), 860-74.

» Geosistemas (1997). Mapa de riesgos naturales en la Argentina. Buenos Aires: Geosistemas.

»Giusti, A. (2007). Censos modernos: 1960, 1970, 1980; 1991, 2001. En S. Torrado (Comp.), Población y bienestar en la Argentina del primero al segundo centenario. Buenos Aires: Edhasa.

» Halperín, L. (1994). Condiciones de vida de la población de Mar del Plata 1992/1994. Mar del Plata: Facultad de Humanidades, UNMP.

" Lo Vuolo, R. y Barbeito, A. (1992). La modernización excluyente. Transformación económica y Estado de Bienestar en Argentina. Buenos Aires: Miño y Dávila.

» Longhi, F., Bolsi, A., Velázquez, G., Paolasso, P. y Celemín, J.P. (2013).

Fragmentación socio-territorial y condiciones de vida en Argentina en los albores del siglo XXI. Revista Latinoamericana de Población,12, 99-132.

» Lucero, P., Mikkelsen, C., Sabuda, F., Ares, S., Aveni, S. y Ondartz, A. (2007). Calidad de vida y espacio: una mirada geográfica desde el territorio local, Hologramática, $1(7), 99-125$.

» Manzano, F. y Velázquez, G. (2016). Calidad de vida y desigualdad en la RMBA. Un análisis a nivel de fracción censal (año 2010). Cardinalis, 6, 19-44.

» McCann, E. (2004). Best Places: Interurban Competition, Quality of Life and Popular Media Discourse. Urban Studies, 41(10), 1909-1929.

» Mikkelsen, C., Ares, S., Sabuda, F. y Lucero, P. (2013). Calidad de vida urbana en la Argentina de la postconvertibilidad. Procesos sociales y territoriales en el período 2003-2012. XII Jornadas Argentinas de Estudios de Población. Bahía Blanca.

» Olave, F. D. y Bodini, H. (1995). Metodología básica para detectar calidad de vida en ciudades intermedias. Depto. de Geografía, Universidad del Bío-Bío.

»Rofman, A. (1999). Desarrollo regional y exclusión social. Transformación y crisis en la Argentina contemporánea. Buenos Aires: Amorrortu.

» Rogerson, R. (1999). Quality of Life and City Competitiveness. Urban Studies, 36(56), 969-985. 
》Santos, M. (1979). O espaço dividido. Os dois circuitos da economia urbana dos países subdesenvolvidos. São Paulo: Hucitec.

"Sterimberg, E., Sánchez, C., Cuervo de Forero, A. y Ramírez, J. (2004). Diseño de un sistema de indicadores socioambientales para el distrito capital de Bogotá. Serie estudios y perspectivas. Santiago de Chile: Cepal - Naciones Unidas.

» Tanguay, G., Rajaonson, J., Lefebvre, J. y Lanoie, P. (2010). Measuring the sustainability of cities: A survey-based analysis of the Use of Local Indicators. Ecological Indicators, 10(2), 407-418.

» Torrado, S. (2007). Población y bienestar. La Argentina del primero al segundo centenario. Buenos Aires: Edhasa.

»Van der Kooy, E. y Pezzella, H. (2013). El gasto en salud en la Argentina. Revista Médicos, 73, 38-45.

»Vega, A., Torcida, S. y Velázquez, G. (2006). Análisis de la Evolución de la Tasa de Mortalidad Infantil en los departamentos de Argentina 1994-2003. Salud Colectiva, 2(3), 237-247.

"Velázquez, G. (2001). Geografía, calidad de vida y fragmentación en la Argentina de los noventa. Análisis regional y departamental utilizando SIG. Tandil: CIG.

"Velázquez, G. (2008). Geografía y Bienestar. Situación local, regional y global de la Argentina luego del Censo de 2001. Buenos Aires: EUDEBA.

》Velázquez, G. (2016). Geografía y calidad de vida en Argentina. Tandil: IGEHCS.

»Velázquez, G. y Celemín, J.P. (2013). La calidad ambiental en la Argentina. Tandil: CIG.

»Velázquez, G., Mikkelsen, C., Linares, S. y Celemín, J.P. (2014). Geografía y calidad de vida en Argentina. Ranking del bienestar por departamentos 2010. Tandil: IGEHCS/CIG.

»Wilkins, H. (2003). The need for subjectivity in ElA: discourse as a tool for sustainable development. Environmental Impact Assessment Review, 23, 401-414.

Guillermo Angel Velázquez / gvelaz@fch.unicen.edu.ar Investigador Superior del CONICET. Director del Instituto de Geografía, Historia y Ciencias Sociales (CONICET/UNCPBA). Publicó 24 libros, 104 artículos en revistas con referato, 42 capítulos en libros de otros autores y 43 ponencias completas. Estos 213 trabajos han sido publicados en libros, revistas y actas de congresos de 27 países. 